Genet. Res., Camb. (1962), 3, pp. 25̃1-272

With 5 text-figures

Printed in Great Britain

\title{
Transfer delay and chromosome withdrawal during conjugation in Escherichia coli
}

\author{
By P. G. DE HAAN* AND J. D. GROSS \\ Medical Research Council Microbial Genetics Research Unit, \\ Hammersmith Hospital, London, W.12
}

(Received 4 October 1961)

\section{INTRODUCTION}

Bacterial conjugation involves random collision of cells of opposite mating type and the formation of effective contact between them, followed by the transfer of genetic material from donor to recipient cell (Hayes, 1953). In $\mathrm{Hfr} \times \mathrm{F}^{-}$crosses, this transfer consists of the progressive penetration of an Hfr chromosome into the $\mathrm{F}^{-}$cell. Penetration starts from the same extremity or origin in all cells of a given Hfr type, and proceeds in a predetermined sequence, transfer of the whole chromosome being completed in approximately 120 minutes (Wollman \& Jacob, 1958; Hayes, 1957). However, spontaneous interruption of conjugation usually occurs before the entire chromosome has been transferred, with the result that zygotes generally receive only a fragment of the Hfr chromosome, the size of this fragment varying from zygote to zygote. The further a genetic marker is from the origin, the lower is its probability of transfer. Transfer has been studied experimentally by stopping conjugation at intervals, either by violent agitation to break up the pairs (Wollman \& Jacob, 1958), or by killing the donor selectively with virulent phage (Skaar \& Garen, 1956; Hayes, 1957). In this way, the order of transfer of markers has been determined and a genetic map constructed in units of time.

Previous studies of the kinetics of transfer were carried out on populations of cells in which effective contact formation and chromosome transfer took place concurrently over almost the entire period of the experiments. Under these conditions the wide spread in the time of formation of effective contacts, and consequently in the time of initiation of chromosome transfer, tends to obscure the finer details of the transfer process. In the present work this complication has been avoided by limiting the period of contact formation to 5 minutes, and following chromosome transfer in the pairs formed during this time. This is done by mixing cultures of the donor and recipient strains at high cell density, leaving them to form contacts for 5 minutes, and then diluting the cells into fresh medium by a factor which is sufficient to prevent the formation of any significant number of new pairs. The kinetics of transfer in the pairs present in the dilute suspension is then studied by interrupting transfer in samples taken at regular intervals, and determining the number of recombinants that are formed from the treated samples.

\footnotetext{
* Present address: Laboratorium voor Microbiologie, State University, Utrecht, Holland.
} 
This procedure conveniently separates chromosome transfer from contact formation, and provides a useful operational definition of the latter process: those pairs which are held together strongly enough to resist diluting are said to have formed effective contact.

The experiments reported here were begun in an attempt to detect any variation among the mating pairs in the time between effective contact formation and the initiation of chromosomal transfer. Such variation has been detected. In some pairs transfer commences very shortly after the formation of effective contact, while in others there is a delay of up to 15 minutes. During the course of experiments designed to yield some information regarding the factors responsible for this variability, marked differences in the extent of chromosome transfer, and in other characteristics of the transfer process, were observed. Under some conditions the number of recombinants in successive blended samples was found to rise to a maximum value and then fall again. The results of an analysis of these phenomena are presented.

\section{Bacterial strains}

\section{MATERIALS AND METHODS}

The Hfr strains employed were:

Hfr $R 1$ and Hfr $R 4$ (spontaneous Hfr mutants isolated from $\mathrm{F}^{+} 58-161: M^{-}$ by Reeves (personal communication)). $M^{+}$derivatives were obtained by transduction with phage $\mathrm{P} 1$.

Hfr H: $B_{1}^{-} \lambda^{-}$(Hayes, 1953; Jacob, Wollman \& Hayes, 1956). A lysogenic derivative obtained by infection with phage $\lambda$ was also used.

HfrP10:TL $L^{-} B_{1}^{-}$, isolated by Jacob \& Wollman (1957).

$\mathrm{Hfr} C: M^{-}$, isolated by Cavalli (1950).

The $\mathrm{F}^{-}$strains employed were:

P678:TL $L^{-} B_{1}^{-} \lambda^{-}$gal $^{-}$(Jacob \& Wollman, 1956). This strain is resistant to phage $\lambda$.

W1:TL $L^{-} M^{-}$and $\mathrm{W} 1: T L^{-} P^{-} B_{1}^{-}$, derivatives of W1:TL- $B_{1}^{-}$(Lederberg \& Lederberg, 1952) were obtained by crossing with derivatives of $\mathrm{HfrC}$.

All Hfr strains were sensitive to streptomycin and phage T6; all $\mathrm{F}^{-}$strains were resistant to both agents.

With the exception of $\mathrm{Hfr} H$ and P678, all strains were lysogenic for prophage $\lambda$.

The origin and direction of transfer of the Hfr strains, as well as the symbols for the genetic markers, are given in Fig. 1.

\section{Media}

Minimal medium: as described by Tatum \& Lederberg (1947), except that asparagine was omitted.

Nutrient broth: $2 \cdot 5 \%$ Oxoid Nutrient Broth No. 2.

Minimal and nutrient agar: made from minimal medium and nutrient broth solidified with $1.5 \%$ and $1.25 \%$ agar respectively. 
EMB medium: as described by Lederberg (1950), with $1 \%$ galactose.

Buffer: an aqueous solution containing $0.4 \% \mathrm{NaCl}, 0.02 \% \mathrm{MgSO}_{4} .7 \mathrm{H}_{2} \mathrm{O}, 0.3 \%$ $\mathrm{KH}_{2} \mathrm{PO}_{4}$ and $0.7 \% \mathrm{Na}_{2} \mathrm{HPO}_{4}$ (pH 7.2 approx.).

Stock cultures were maintained at $4^{\circ} \mathrm{C}$. on Dorset egg slopes.

Overnight cultures were prepared by inoculating $5 \mathrm{ml}$. of nutrient broth or appropriately supplemented minimal medium, and incubating for about 18 hours at $37^{\circ} \mathrm{C}$. without aeration. Fresh cultures were obtained by diluting the overnight cultures tenfold into $5 \mathrm{ml}$. of fresh medium in screw-capped bottles and incubating for the desired period on an inclined turntable rotating at approximately 33 r.p.m. When cells starved of their required amino-acids were required, a fresh, minimal

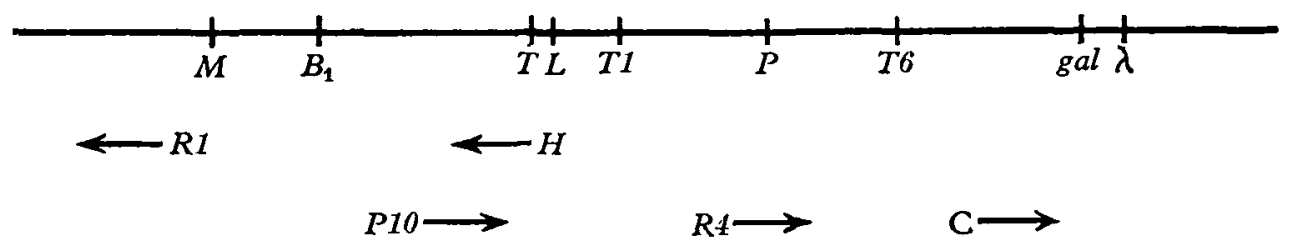

Fig. 1. The order of various loci on the Escherichia coli K-12 chromosome. The relative positions of the loci are only approximate. The region shown constitutes about one-third of the chromosome. The position of the origin and the direction of transfer of the Hfr strains employed (see text for symbols) are indicated by the arrows. The symbols of the markers represent:

$$
\begin{aligned}
& M=\text { methionine } \quad \mathrm{Tl}=\text { phage } \mathrm{Tl}\} \text { Resistance }(R) \text { or } \\
& \left.B_{1}=\text { vitamin } B_{1} \quad T 6=\text { phage } \mathrm{T} 6\right\} \text { Sensitivity }(S) \\
& T=\text { threonine }\} \text { synthesis } \quad g a l=\text { galactose fermentation } \\
& L=\text { leucine } \quad \lambda=\text { lysogenicity }\left(^{+}\right) \text {or non-lysogenicity }\left(^{-}\right) \\
& P=\text { proline for phage } \lambda
\end{aligned}
$$

The markers $T$ and $L$ are used throughout as one marker, $T L$.

grown, culture was centrifuged and washed three times and resuspended in minimal medium with the appropriate amino-acid(s) omitted. The suspension was then incubated on the turntable for a period of 2 hours. In all experiments $0.2 \mathrm{ml}$. of a fresh ( $1 \frac{1}{2}$ hour) culture of the Hfr strain was mixed with $1 \mathrm{ml}$. of an overnight culture of the $\mathbf{F}^{-}$strain, and $1 \mathrm{ml}$. of fresh medium, to give a final concentration of about $2 \times 10^{7} \mathrm{Hfr}$ and $4 \times 10^{8} \mathrm{~F}^{-}$cells per $\mathrm{ml}$. The mixture of $\mathrm{Hfr}$ and $\mathrm{F}^{-}$cells was rotated for 5 minutes and then diluted gently by a factor of 500-1000 into the desired pre-warmed medium to prevent further contact formation. This diluted suspension was not rotated. At regular intervals samples were withdrawn and transfer interrupted by one of the following methods:

1. The samples were agitated violently for 30 seconds on a microid flask shaker (Griffin \& George Ltd.) at maximum speed, to break up the mating pairs. This method will be referred to as 'blending' since it is used for the same purpose as the Waring Blendor technique employed by Wollman \& Jacob (1958).

2. Phage $\mathrm{T} 6$, to which the Hfr but not the $\mathrm{F}^{-}$strain is sensitive, was added to the samples to give a final concentration of about $10^{10}$ particles per $\mathrm{ml}$. The phage 
lysates were irradiated with ultraviolet light to a survival of $0.1 \%$ plaque forming units since the lysates appeared to contain host range mutants which could multiply in the T6-resistant $\mathrm{F}^{-}$cells and destroy some recombinants.

The number of recombinants for auxotrophic characters was determined by plating duplicate $0.1 \mathrm{ml}$. samples onto minimal agar lacking the appropriate growth factor. $\mathrm{Gal}^{+}$recombinants were selected on EMB galactose plates, and also on plates with appropriately supplemented minimal medium in which glucose was replaced by galactose as sole carbon source. All plates contained $100 \mu \mathrm{g}$. per ml. streptomycin to kill the Hfr cells. When samples from mating suspensions in minimal medium were plated, $0.1 \mathrm{ml}$. of broth was added so that the postzygotic environment of the cells should be the same as for the suspensions in brotb. It has been found (Gross, unpublished) that the efficiency of recombinant formation in zygotes derived from broth-grown $\mathrm{F}^{-}$cultures is 2-5 times greater when the zygotes are incubated in the presence of broth than when no broth is present. This effect of broth is restricted to approximately the first hour after zygote formation, the environment to which the zygotes are thereafter exposed having no significant effect on the yield of recombinants. Samples were plated soon after treatment since new contacts begin to form after about 40 minutes, particularly in broth, due to cell multiplication and the consequent increase in the probability of collision between cells of opposite mating type. Zygotic induction was measured by the method of Jacob \& Wollman (1956) using a streptomycinresistant derivative of strain $\mathrm{C} 600$ as indicator.

All experiments were carried out in a constant-temperature room maintained at $37^{\circ} \mathrm{C}$.

\section{RESULTS}

\section{Delay in the initiation of transfer}

If the interval between effective contact formation and the initiation of chromosomal transfer is the same for all Hfr cells, and if the rate of transfer is also the same, then, owing to the oriented nature of the transfer process, the interval between the time when any given marker is first transferred to $\mathrm{F}^{-}$cells and the time when all transfer of that marker has taken place should be no greater than the period allowed for effective contact formation. If, however, there is any variation in either the time of onset of transfer or the rate of transfer, then the transfer of the marker will be spread over an interval greater than that allowed for contact formation. The two sources of asynchrony can be distinguished experimentally by following the transfer of two well-spaced markers. If asynchrony is due solely to a variable delay in the initiation of transfer then the spread for the two markers should be the same, whereas if there is any variation in the rate of transfer the spread for the marker entering later should be greater than for the earlier one.

Fig. 2 presents the results of an experiment with $H f r R 4$ in which the transfer in broth of the markers $T L^{+}$and $M^{+}$was followed in pairs formed during a 5-minute period. Transfer of these markers is separated by 20 minutes, $T L^{+}$recombinants 
being first found in samples blended at 10 minutes, while the first $M^{+}$recombinants occur in samples blended at 30 minutes. The fact that more than twenty times as many $T L^{+}$recombinants as $M^{+}$recombinants were formed is due to spontaneous interruption of conjugation during the transfer of the chromosomal segment between the two markers. It may be seen that for both the $T L^{+}$and the $M^{+}$

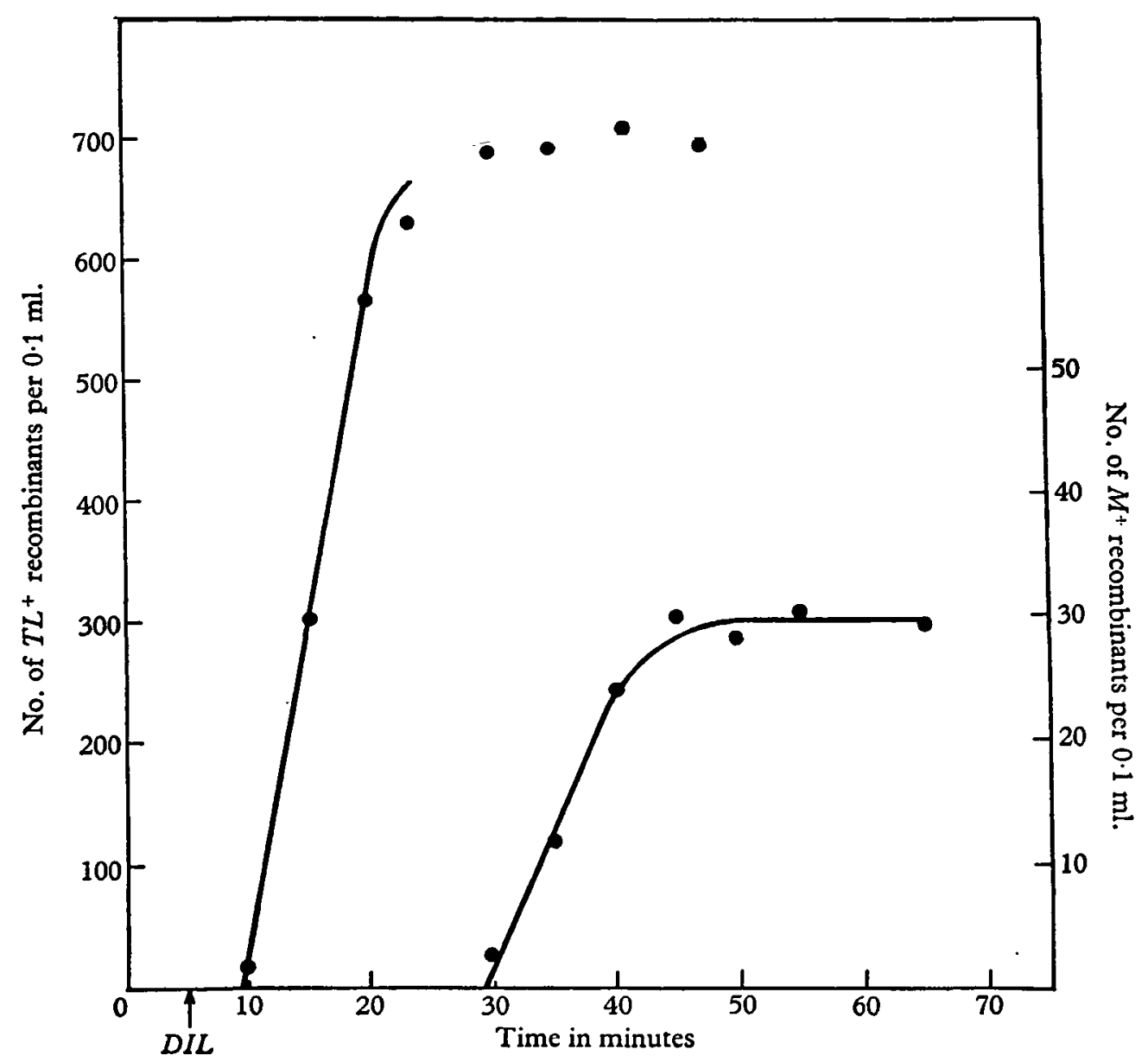

Fig. 2. Trangfer curves for the $T L^{+}$and $M^{+}$markers in a cross between prototrophic Hfr $R 4$ and $F^{-}: T L-M-$. Broth-grown cultures were mixed for 5 minutes and diluted 500-fold into prewarmed broth at the time indicated by the arrow. Transfer was interrupted at intervals by blending. Note the difference in scale for $T L^{+}$and $M^{+}$recombinants.

markers there was an interval of about 20 minutes between the time of blending at which the first recombinants were recovered and the time when transfer was completed. This result indicates that there is an asynchrony of about 15 minutes over and above that introduced by the period of contact formation. Further, the fact that the degree of asynchrony is the same for the two markers shows that 
there is no detectable contribution from variation in the rate of transfer, and that the observed spread is therefore due to a variable delay between the time of effective contact formation and the onset of chromosomal transfer.

However, the absence of variation in the rate of transfer by different cells which applies to Hfr $R 4$ does not hold for all donor strains, e.g. for Hfr $H$ or HfrR1. This may be seen from the results for crosses involving $\mathrm{Hfr} H$ which are presented in Fig. 3 and which will be discussed in greater detail below. The curve for transfer of $T L^{+}$in the cross with $\mathrm{Hfr} H\left(\lambda^{-}\right)$rises until 30 minutes, indicating a spread of about 20 minutes as in the case of HfrR4, whereas the spread for $\lambda$ prophage or $\mathrm{gal}^{+}$is about 35 minutes. The greater spread for the later markers is taken to indicate the existence of variation in the rate of transfer. This variation is sufficient to account for the transfer spread observed with $\mathrm{Hfr} H$ without the necessity of assuming variation in the time of initiation of transfer such as has been deduced from the results with $\mathrm{Hfr} R 4$. However, even in the case of HfrR4, and of $\mathrm{HfrC}$ which behaves in the same way, a quite different explanation for the spread in the transfer curves must be considered. In experiments on the kinetics of transfer of a prototrophic or other typical genetic marker the extent of transfer of that marker at successive intervals is estimated indirectly by determining the number of recombinants formed from blended samples. It is implicitly assumed that the number of recombinants formed depends only on the number of zygotes which have received the marker in question, i.e. that the probability of recombinant formation from such zygotes is independent of the time of blending. However, at early times of blending those zygotes which have received the selected marker will contain segments of the Hfr chromosome which extend only a short distance beyond the region of the selected marker. As the time before blending increases, the length of the fragment distal to the selected marker will progressively increase due to penetration of an additional length of chromosome in the intervening time. If the probability that a marker will be incorporated into a recombinant depends on the distance between it and the end of the fragment, then this probability will be small for the zygotes contained in the samples blended at early times and will increase as the time elapsing before blending increases. In that case, the spread in the curves of Fig. 2 could be due to a progressive increase in the probability of recombinant formation in a constant number of zygotes. The selected marker would have penetrated into all of these zygotes within a 5-minute period and there would be no need to infer the existence of any spread in the actual time of transfer of that marker.

Observation of the transfer of prophage $\lambda$ may be used to test this explanation, since evidence has been presented (Jacob \& Wollman, 1956) that in crosses between lysogenic $\mathrm{Hfr}$ and non-lysogenic $\mathrm{F}^{-}$the prophage is induced as soon as it enters the zygote in virtually all cases. It is therefore possible to follow directly the transfer of the prophage locus without the necessity of formation of genetic recombinants, by measuring the production of infective centres. If the spread in the transfer curves of Fig. 2 were due to an effect of fragment length on the probability of recombinant formation, then no such spread should be observed 
for the transfer of prophage $\lambda$. If, on the other hand, the spread is really due to asynchronous transfer resulting from a variable delay before the initiation of transfer, then a spread should be observed. Unfortunately, Hfr $H$ is the only donor strain available to us with which zygotic induction can be conveniently studied, and it gives rise to a transfer spread due to variability in the rate of transfer by different donor cells. The asynchrony in the transfer of prophage due to this cause makes it impossible to determine whether there is any additional spread due to variable delay in the initiation of transfer. Therefore a rather more elaborate test must be applied to determine whether the spread in the curves obtained with $\mathrm{Hfr} R 4$ could be due to an effect of fragment length on the probability of recombinant formation. This approach involves determining whether the kinetics of transfer of prophage $\lambda$ is the same as that for a very closely linked marker which requires recombination to take place before it can be detected. For this purpose the transfer of $\lambda$ prophage by $\mathrm{Hfr} H\left(\lambda^{+}\right)$has been compared with the transfer of the very closely linked $g a l^{+}$marker in a parallel experiment with Hfr $H\left(\lambda^{-}\right)$in which no zygotic induction can occur. The transfer of the $T L^{+}$ marker was followed in both experiments and was used as a standard of comparison between them. If an effect of fragment length exists, then it will be superposed on the spread for the $g a l^{+}$marker, but not on the spread for $\lambda$ prophage, and the curve for transfer of $\mathrm{gal}^{+}$should be displaced to the right of that for $\lambda$ prophage. If no such effect exists, the curves should be parallel.

The results of a comparison of this type are given in Fig. 3, from which it may be seen that the two curves are in fact parallel. Prophage $\lambda$ and the $g \mathrm{l}^{+}$marker begin to enter zygotes at about 27 and 25 minutes respectively, and reach a plateau shortly after 60 minutes. The rise in number of infective centres after 75 minutes is due to lysis and release of $\lambda$ from zygotes in which induction and phage development has occurred. The close parallelism of the two curves indicates that, within the accuracy of the experiment, the length of fragment distal to a selected marker has no effect on the probability that the marker will be incorporated into a recombinant. It has been shown (Gross \& de Haan, unpublished) that the probability of obtaining a recombinant which carries the proximal but not the distal of two closely linked markers is greater when the transferred chromosomal fragment terminates between the two markers than when it overlaps them both. This result indicates that there is a high probability of crossing-over at the distal end of the fragment. This could account for the lack of dependence of the probability of incorporation of a marker on its distance from the end of the transferred fragment. At all events the results presented in Fig. 3 show that the spread in the transfer curves obtained with $\mathrm{Hfr} R 4$ cannot be due to a progressive increase in the probability of recombinant formation, but must be due to variable delay before the initiation of transfer. Some Hfr cells commence transfer shortly after forming effective contact, while in others there is a delay of up to 15 minutes before the start of transfer. Presumably this variable delay also occurs in the case of $\mathrm{HfrH}$ and other strains showing variation in the rate of transfer but cannot be demonstrated owing to the existence of the latter variation. 
Several other points may be mentioned in connection with the experiment of Fig. 3. The kinetics of transfer of $\lambda$ prophage as measured by the progressive rise in number of infective centres may also be inferred from the curve for transfer of

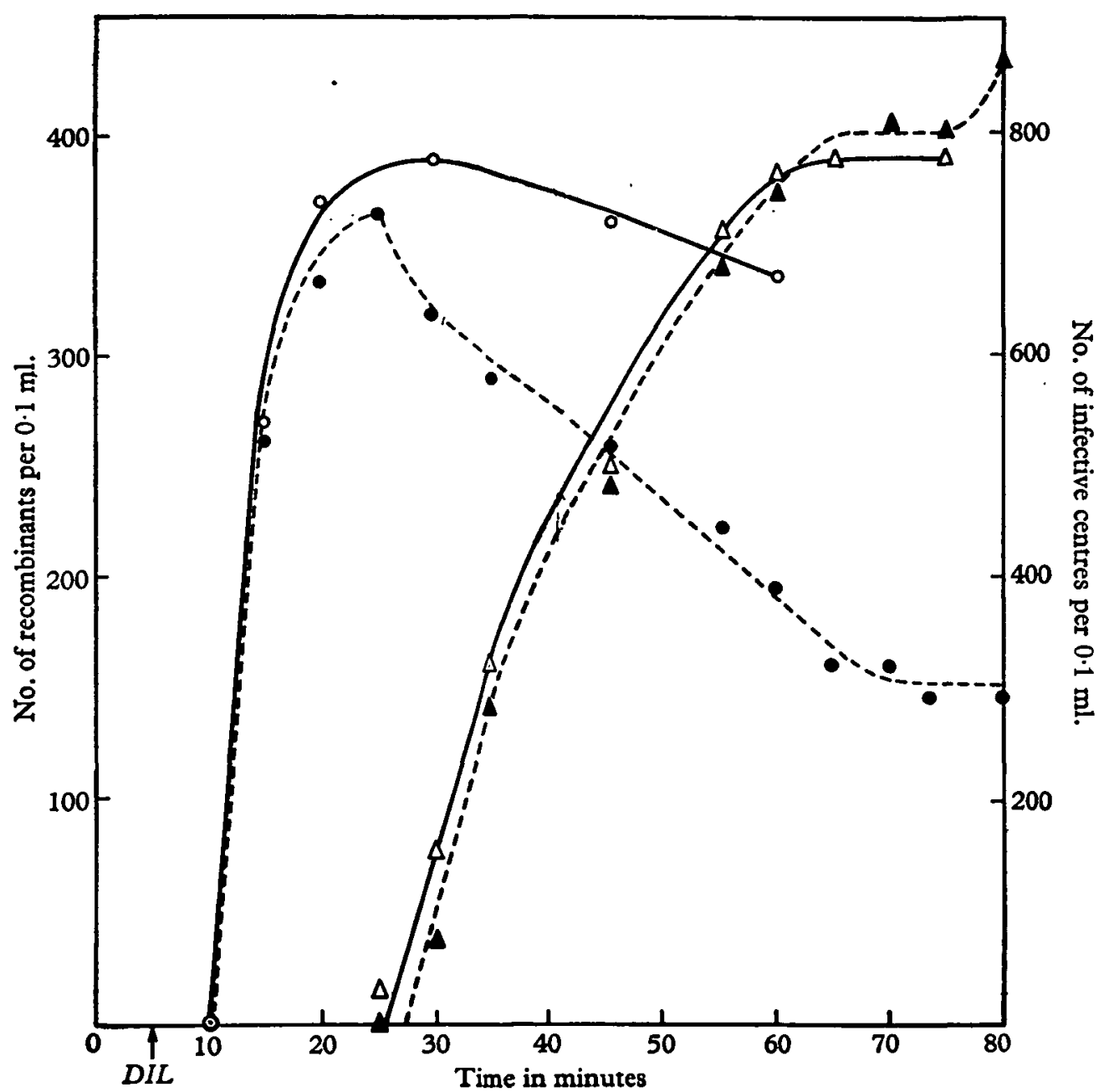

Fig. 3. Transfer curves for the $T L^{+}$and gal ${ }^{+}$markers, and for prophage $\lambda$ in crosses between $\mathrm{Hfr} H$ and P678. Cultures were grown and mixed in broth. Five minutes later (indicated by the arrow) the mixtures were diluted 1000 -fold into minimal medium, and samples blended at intervals. The curves in solid lines are from a cross with the non-lysogenic HfrH $\left(0=T L^{+}\right.$recombinants; $\Delta=g \mathrm{gl}^{+}$ recombinants) while the dotted curves are from the experiment with the lysogenic Hfr $H\left(\bullet=T L^{+}\right.$recombinants; $\Delta=$ infective centres). Note the difference in scale between left and right ordinate.

$T L^{+}$in the cross with $\mathrm{Hfr} H\left(\lambda^{+}\right)$. This rises at first just like the corresponding curve for $\mathrm{Hfr} H\left(\lambda^{-}\right)$, but thereafter there is a sharp fall, the number of $T L^{+}$ recombinants levelling off finally at about 60 minutes, by which time the number of $\mathrm{TL}^{+}$recombinants recovered is less than half the number recovered at 25 
minutes. This fall, which is much more marked than in the curve for $\mathrm{Hfr} H\left(\lambda^{-}\right)$, results from the destruction of zygotes which would otherwise give rise to $\mathrm{TL}^{+}$ recombinants, due to transfer of $\lambda$ prophage and consequent zygotic induction. When conjugation is interrupted by blending at 25 minutes no transfer of $\lambda$ prophage has yet taken place, but during the next 35 minutes it is transferred to a progressively larger number of zygotes, leading to their destruction (Wollman \& Jacob, 1958). The survival of some $T L^{+}$recombinants at 60 minutes when transfer of $\lambda$ is completed is due to spontaneous interruption of conjugation during transfer of the chromosomal segment between $T L^{+}$and $\lambda$ prophage. Interruption must occur in about $50 \%$ of the zygotes to account for the proportion of $T L^{+}$recombinants which survive. The fact that the number of $\mathrm{gal}^{+}$recombinants is as great as the number of $T L^{+}$recombinants, despite the breakage in the interval between the two markers, is explained by differences in the efficiency of genetic integration on the media employed for enumerating $T L^{+}$and $g a l^{+}$recombinants. The curve for $\mathrm{gal}^{+}$recombinants shown in Fig. 3 is derived from counts on EMB galactose medium which is rich in nutrients, and on this medium the efficiency of genetic integration is about twice as great as on minimal medium, on which the $T L^{+}$ recombinants were measured. In fact $\mathrm{gal}^{+}$recombinants were also counted on minimal medium, and the number recovered was always slightly less than half the number on EMB. This is the value which should be compared with the number of $T L^{+}$recombinants, and it is in good agreement with the estimate of breakage in the interval between the two markers.

The actual efficiency of integration, i.e. the probability that a zygote which has received a given marker will give rise to a recombinant carrying that marker, may be calculated in the case of the $\mathrm{gal}^{+}$marker in a manner analogous to that employed by Wollman \& Jacob (1959). The number of infective centres in the cross with $\mathrm{Hfr} H\left(\lambda^{+}\right)$may be taken as a measure of the number of zygotes which received the $\mathrm{gal}^{+}$marker in the cross with $\mathrm{Hfr} H\left(\lambda^{-}\right)$, since the site of $\lambda$ prophage and the $\mathrm{gal}^{+}$marker are very closely linked and since the initial equality in the number of $T L^{+}$recombinants indicates that equal numbers of mating pairs were involved in the two crosses. Taking account of the difference in scale, it may be seen that there are twice as many infective centres as $\mathrm{gal}^{+}$recombinants. Therefore, half of the zygotes which received the $\mathrm{gal}^{+}$marker gave rise to $\mathrm{gal}^{+}$recombinants and the efficiency of integration is 0.5 . This applies only to samples plated on EMB. On minimal medium, as noted, the efficiency is about halved.

\section{Stability of effective contact between mating pairs}

There are two differences of some interest between the results of the experiment of Fig. 2, which was performed in broth, and that of Fig. 3, performed in minimal medium.

1. In the cross with $\mathrm{Hfr} H\left(\lambda^{-}\right)$in which no zygotic induction can occur, there is nevertheless a gradual fall in the number of $T L^{+}$recombinants after 30 minutes, whereas in the cross of Fig. 2 there is no such fall in the curves for either the $T L^{+}$ or the $M^{+}$recombinants. 
2. Transfer of markers some distance from the origin occurs with much higher efficiency in the cross with Hfr $H$ than with HfrR4. Although the distance between $\mathrm{gal}^{+}$and $T L^{+}$is roughly the same as the distance between $M^{+}$and $T L^{+}$, as measured by the time required to transfer these segments in the two crosses, there are half as many $g a l^{+}$as $T L^{+}$recombinants in the former cross, but only onetwentieth as many $M^{+}$as $T L^{+}$in the latter.

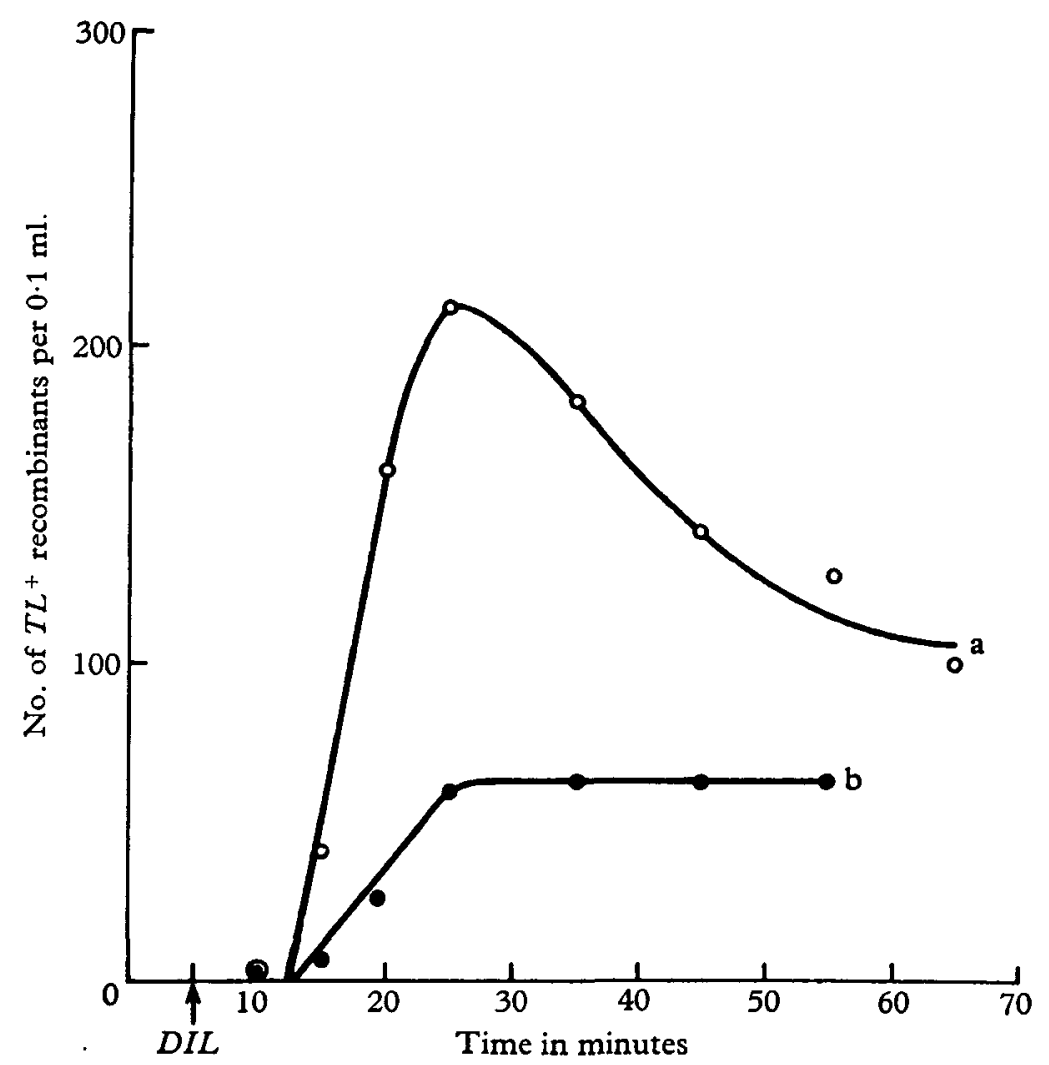

Fig. 4. Comparison of the transfer of the $T L^{+}$marker in broth and in minimal medium in a cross between Hfr $R 4: M^{-}$and $\mathrm{F}^{-}: T L^{-} M^{-}$. Cultures were grown and mixed in broth. Five minutes later the mixture was diluted 500 -fold into minimal medium (curve $a$ ) and broth (curve $b$ ), and samples were blended at intervals.

Some understanding of these differences may be gained by a comparison of transfer in broth and minimal medium. Fig. 4 presents the results of an experiment with Hfr $R 4$ in which broth-grown cells were mixed for the usual period and then diluted into broth and into minimal medium, and the kinetics of transfer of the $T L^{+}$marker in the two media compared. It may be seen that in both media the first $T L^{+}$recombinants are found at 10 minutes, and thereafter increase progressively in number until 25 minutes. However, the number of recombinants in broth (curve $b$ ) is considerably less than in minimal medium (curve $a$ ) and, whereas in broth the number remains constant after 30 minutes, in minimal medium there 
is a sharp fall in numbers in successive blended samples. The number in minimal medium stabilizes finally at a value roughly twice that obtained in broth.

There is good evidence that the greater number of recombinants formed in minimal medium compared to broth is due to transfer of the $T L^{+}$marker to a greater number of zygotes in the former medium, and not to some effect of differences in the environment acting after transfer has taken place. It cannot be due to a greater efficiency of recombinant formation in minimal medium, since the presence of broth in fact raises this efficiency. Furthermore, it may be recalled that broth is routinely added at the time of plating to samples taken from minimal medium. Any unfavourable effect of broth during blending or indeed at any time subsequent to transfer is excluded by the fact that the addition of concentrated broth immediately before blending to samples taken from minimal medium does not reduce the yield of recombinants. Furthermore, as will be described below, the extent of the difference between the number of recombinants formed in broth and in minimal medium depends on the Hfr strain employed, but not at all on the recipient strain. This indicates that it is the activity of the Hfr, i.e. transfer, that is involved, and not processes which take place in the $\mathrm{F}^{-}$cells after transfer.

Experiments in which concentrated broth is added to the minimal medium at various times after mixing show that the presence of broth affects the transfer of the $T L^{+}$marker during the first 30 minutes after mixing. If the cells are diluted into minimal medium to which broth has been added, a curve of type $b$ (Fig. 4) is obtained, typical of transfer in the presence of broth. The addition of broth at progressively later times gives rise to a series of curves intermediate between $a$ and $b$. However, if the addition of broth is delayed until $\mathbf{3 0}$ minutes after mixing, then it no longer has any effect on the transfer of the $T L^{+}$marker, though it has a pronounced effect on the transfer of the $M^{+}$marker. Thus the drop in the number of $T L^{+}$recombinants in samples blended at intervals after 30 minutes occurs whether or not broth is added to the medium at this time. It is found that the rate of fall is in fact somewhat accelerated by the presence of the broth, but the final extent of the fall is unaltered.

It appears, therefore, that the presence of broth in the medium has two effects on the transfer curve for the $T L^{+}$marker: (1) the addition of broth at any time during the first 30 minutes after mixing reduces the final number of $T L^{+}$recombinants obtained; the reduction is greater, the earlier the time of addition of the broth; (2) if broth is present throughout the first 30 minutes after mixing there is no subsequent fall in the number of $T L^{+}$recombinants.

The restriction of the period of effectiveness of broth with respect to the transfer curve of the $T L^{+}$marker to the first 30 minutes after mixing is in good agreement with the evidence that this is the period during which transfer of the $\mathrm{TL}^{+}$marker takes place, the spread being due to the variation in the time of initiation of chromosomal transfer. By 30 minutes all transfer of the $T L^{+}$marker which can occur has been completed. The effect of broth on the final yield of $T L^{+}$recombinants is then due to a reduction in the number of pairs in which transfer of the $T L^{+}$marker takes place. On the other hand, the fall in number of $T L^{+}$recombi- 
nants commences after transfer of the $T L^{+}$marker has been completed. It is important to note, however, that this fall by its very nature depends on the mating pairs remaining united, since it is detected experimentally as a reduction in the number of recombinants in samples blended at successive intervals to separate the pairs.

The two effects of broth, the first on the extent of transfer of the $T L^{+}$marker, and the second on the fall in number of recombinants after transfer has been completed, can be related to one another by the hypothesis that the presence of broth reduces the stability of the mating pairs. If this were the effect of broth, the extent of transfer of any marker would be reduced, since a high proportion of pairs would separate before transfer of that marker could take place. The absence of a fall subsequent to transfer would be accounted for at least in part by the fact that by the time transfer of the marker in broth was completed very few pairs would remain intact, and since the fall is due to some phenomenon which occurs only in intact pairs little or no fall would be observed. In minimal medium, on the other hand, the pairs would be considerably more stable, so that any given marker would be transferred to a greater extent, and when its transfer was completed a large number of pairs would remain intact with the result that the process leading to the fall could take place.

If this explanation is correct, then the rate of separation of pairs in broth must be such that very few remain intact by 30 minutes after mixing. In that case, markers entering some time after $T L^{+}$should be hardly transferred at all in broth, and in general the gradient of transfer of markers with increasing distance from the origin should be much steeper in broth than in minimal medium. Now, it may be recalled that it is precisely the absence of a drop in number of recombinants, and the much less frequent transfer of later markers, which differentiates the results of the experiment with $R 4$ in broth (Fig. 2) from those obtained with $\mathrm{Hfr} H$ in minimal medium (Fig. 3). The results of a comparison of the relative frequency of transfer of the $P, T L$ and $M$ markers by Hfr $R 4$ in broth and in minimal medium are given in Table 1 . It may be seen that the gradient of transfer of these markers is very much steeper in broth than in minimal medium, and the $M^{+}$marker is transferred thirty times more frequently in minimal medium than in broth. These results strongly support the hypothesis that the presence of broth reduces the stability of mating pairs. Analysis of the results in Table 1 (Symonds, 1962) suggests that the rate of separation of pairs in broth but not in minimal medium may increase as a function of time after contact formation, since the gradient of transfer in broth does not appear to be a simple exponential function of distance from the origin. This suggestion is strikingly corroborated by the fact that a small but clear drop was observed in the broth curve for the $P^{+}$marker but not for the $\mathrm{TL}^{+}$or $\mathrm{M}^{+}$markers. Owing to the greater stability of the pairs during the first minutes after contact formation, a sufficient number of intact pairs in which the $P^{+}$marker had entered the $\mathrm{F}^{-}$accumulates to give rise subsequently to a significant drop.

Table 2 gives the results of a similar comparison for $\mathrm{Hfr} R 1$ which transfers the 
$T L^{+}$and the $M^{+}$markers in the reverse order. It may be seen that in this case the presence of broth has little effect on the gradient of transfer which is similar to that of HfrR4 transferring in minimal medium, indicating that the stability of the contacts formed by $\mathrm{Hfr} R 1$ is high irrespective of the presence or absence of

Table 1. Relative frequency of marker transfer in broth and minimalmedium by $\mathrm{Hfr \textrm {R } 4}$

Number of recombinants $/ 0.1 \mathrm{ml}$.

Transfer in minimal medium

Transfer in broth

Relative frequency of recombinants $\ddagger$

Minimal medium

Broth

No. of recombinants in minimal medium $\ddagger$

No. of recombinants in broth

Time of entry (minutes)

Selected recombinant class

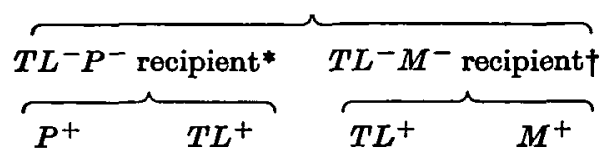

$\begin{array}{crrr}493 & 364 & 342 & 149 \\ 267 & 129 & 691 & 30 \\ P^{+} & & T L^{+} & M^{+} \\ & & & \\ 100 & & 74 & \\ 100 & 48 & 28 \\ 1.8 & 2 \cdot 8 & 28 \cdot 6 \\ 4 & 10 & 27 \\ \text { tudied in the same experiment. } \\ \text { tudied in separate experiments. } \\ \text { recombinants in the different experiments. }\end{array}$

broth. If the fall in number of recombinants subsequent to transfer depends, as suggested, on the stability of mating pairs, then one would expect that in the case of HfrR1 there should be a fall in broth as well as in minimal medium, and this is indeed the case. It may be mentioned that $\mathrm{Hfr} H$ is actually like $\mathrm{Hfr} R 1$ in that it

Table 2. Relative frequency of marker transfer in broth and minimal medium by $B f r \mathrm{R} 1$

\begin{tabular}{lrrr} 
& \multicolumn{2}{c}{ No. of recombinants $/ 0 \cdot 1 \mathrm{ml} .^{*}$} & No. of $M^{+}$recombinants \\
\cline { 2 - 3 } & $M^{+}$ & $T L^{+}$ & No. of $T L^{+}$recombinants \\
Transfer in minimal medium & 550 & 244 & $2 \cdot 2$ \\
Transfer in broth & 412 & 106 & $3 \cdot 9$ \\
Time of entry (minutes) & 9 & 24 &
\end{tabular}

* Transfer in broth and in minimal medium studied in the same experiment.

is relatively insensitive to broth and gives the fall in both media. Of six Hfr strains so far tested, four, $\mathrm{Hfr} R 4, \mathrm{Hfr} P 10, \mathrm{Hfr} C$ and $\mathrm{Hfr} R 3$, are highly sensitive to broth, while $\mathrm{Hfr} H$ and Hfr $R I$ are not. Since no effect of varying the recipient strain has been observed it would appear that the stability of the contact between Hfr and $\mathrm{F}^{-}$depends only on the activity of the Hfr cells. 
The effect of broth is not due to the provision of growth factors required by the $\mathrm{Hfr}$, since the addition of these factors to the minimal medium, or the substitution of a prototrophic Hfr ( $\mathrm{Hfr} R 4 M^{+}$or HfrP10TL $L^{+}$) does not alter the transfer curve, and therefore can have no effect on the stability of the pairs. However, the addition to the minimal medium of vitamin-free casamino acids at a concentration as low as $0.1 \%$, or of an equivalent mixture of amino-acids, results in transfer curves similar to those obtained in broth. Experiments in which various amino-acids were omitted from the mixture indicated that all must be present to give this effect.

\section{Chromosome withdrawal}

There is thus good evidence that the effect of the presence of broth may be ascribed to the action of a complete mixture of amino-acids in stimulating the activity of the cells of certain Hfr strains in such a way as to reduce the stability of the mating pairs formed by them. This explanation accounts in a unitary fashion for both the lower extent of chromosomal transfer and the absence of a fall subsequent to transfer which characterizes transfer by these strains in broth. However, no explanation has yet been given for the fall itself. It was pointed out above that the event which is responsible for the fall, whatever its nature, must occur only in the mating pairs, since it is prevented by separating the pairs. That only the activity of the Hfr cells is implicated is suggested by the fact that the same drop in the number of $T L^{+}$recombinants after 30 minutes is observed in a cross of the type illustrated in Fig. 4 if the $\mathrm{F}^{-}$is starved of its required aminoacids throughout the experiment. With $\mathrm{Hfr} R A$ a fall similar to that for the $T L^{+}$ marker is also observed after transfer of the $P^{+}$and $M^{+}$markers in minimal medium, indicating that the fall cannot be due to transfer of some lethal factor. This view is supported by the fact that the fall may be observed in crosses involving any $\mathrm{Hfr}$ and any $\mathrm{F}^{-}$strain, provided that the right experimental conditions are employed.

We propose that the fall is due to the withdrawal in some of the mating pairs of the segment of the Hfr chromosome which has been transferred to the $\mathrm{F}^{-}$cells. Withdrawal is assumed to occur with a certain probability at any time after the commencement of chromosomal transfer. But, since it is detected experimentally as a fall in the number of recombinants for a given marker, it only becomes apparent when the reduction in number of recombinants in successive blended samples due to withdrawal from $\mathrm{F}^{-}$cells which have already received the marker exceeds the increase due to additional transfer. During the initial period of transfer of any marker the rate of transfer exceeds withdrawal, otherwise no transfer would ever be observed, but as time progresses the effect of withdrawal begins to predominate. It is clear, therefore, that the phenomenon of withdrawal would be virtually imperceptible unless the period of effective contact were restricted, since the wide spread in the time of transfer would obscure the withdrawal. The absence of any fall in broth in crosses involving strains sensitive to the effect of broth follows clearly from these considerations, since by the time transfer is approaching completion too few pairs remain intact for any withdrawal 
to be observed. It should, however, be mentioned that in order to reconcile this explanation of the absence of any fall in broth with the fact that the spread in the transfer curve is as great as in minimal medium, it is necessary to assume that in addition to the rate of separation of mating pairs being increased in the presence of broth, the probability of chromosome withdrawal is reduced (Symonds, 1962).

One important condition necessary for the detection of withdrawal has not yet been mentioned: the treatment employed to interrupt transfer (and hence to follow its progress) must also prevent withdrawal. Treatment by blending fulfils this condition since it physically separates the donor and recipient cells. Consequently, when successive samples are blended at intervals after the completion of transfer, withdrawal is interrupted at intermediate stages before its completion, and a progressive fall in number of recombinants is observed. If, however, a treatment were employed which stopped transfer but did not prevent withdrawal, then all samples taken after transfer of the marker under examination was completed should yield the same number of recombinants, since these would derive only from those zygotes left after all withdrawal had taken place. Consequently, no fall in the number of recombinants would be observed. Experiments in which transfer is interrupted by treatment of the suspension with phage T6, to which only the $\mathrm{Hfr}$ is sensitive, give quite a different result from the experiments employing the blending technique, and lend strong support to the idea of chromosomal withdrawal. In addition, they provide convincing evidence that broth decreases the stability of mating pairs.

The results of a comparison of blending and $\mathrm{T} 6$ treatment are given in Fig. 5 . Considering first the curves obtained for transfer in minimal medium, it may be seen that the time at which the first recombinants are formed in the T6 curve is the same as in the curve obtained by blending. This shows that $\mathrm{T} 6$ stops transfer rapidly since otherwise recombinants would appear earlier than with blending. The curve obtained by $\mathrm{T} 6$ treatment differs from that obtained by blending in two major respects: (1) the number of recombinants in samples treated with T6 is consistently less than in samples blended at the same time, and (2) whereas the blended curve shows the accustomed fall after $\mathbf{3 0}$ minutes, there is no fall in the T6 curve. Instead the number of recombinants formed from successive samples levels off towards 30 minutes and thereafter remains constant at a value similar to that for the recombinants formed in the blended curve when the fall appears to be approaching completion.

These differences are easily accounted for if it is assumed that adsorption of T6 to an Hfr cell which is in process of transferring to its $\mathbf{F}^{-}$partner arrests the transfer but does not prevent subsequent withdrawal. If this is so, the recombinants formed from a T6-treated sample should derive only from those zygotes from which the Hfr segment bearing the selected marker is not ultimately withdrawn. The number of recombinants formed from samples treated with T6 should be less than in blended samples, since withdrawal can occur in the former, but not in the latter. Similarly, there should be no fall in the T6 curve for the reasons 
already discussed. Furthermore, the constant yield of recombinants in all samples treated with $\mathrm{T} 6$ after 30 minutes should equal the yield in the sample blended after sufficient time has elapsed before blending for essentially all withdrawal to have occurred, and this is seen to be the case. It is worth noting that in principle

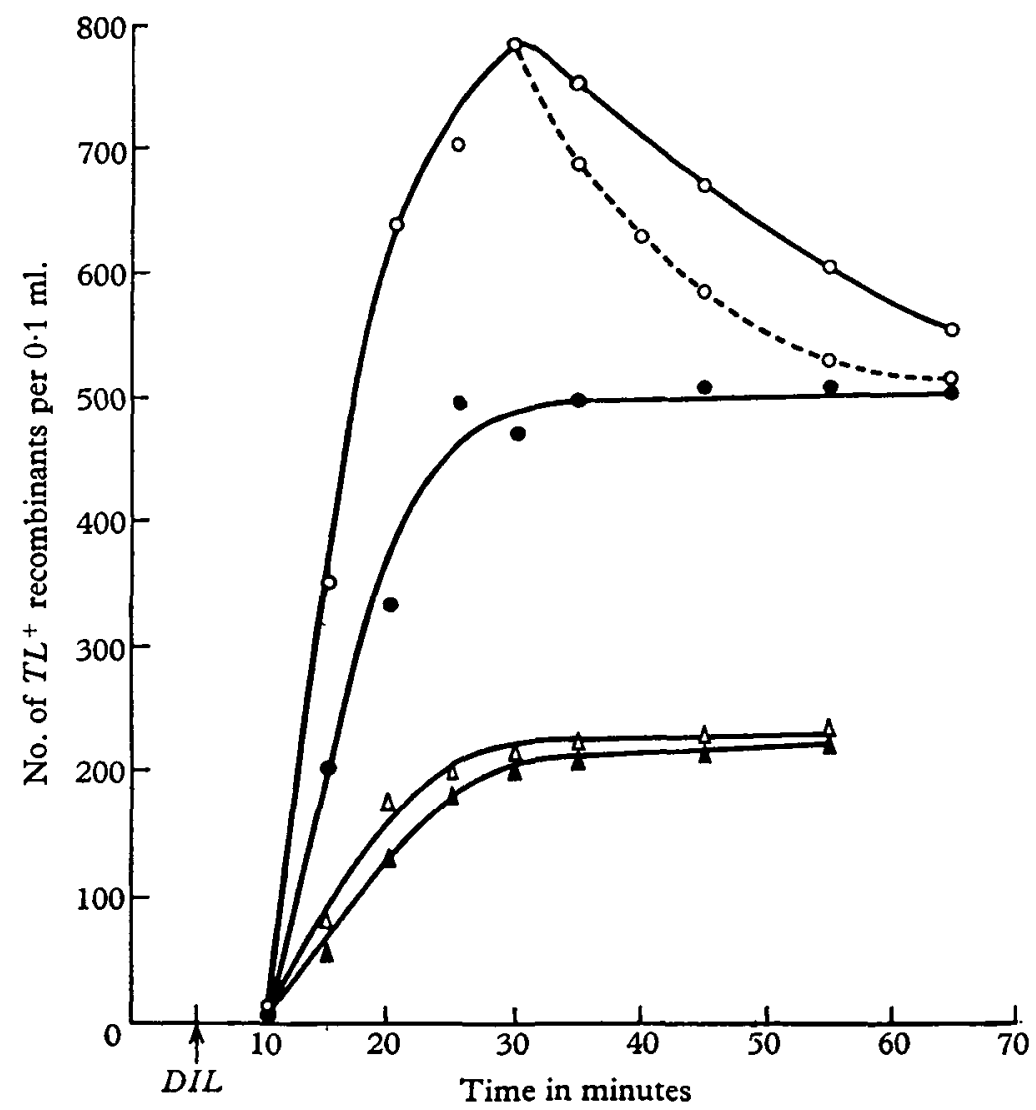

Fig. 5. Comparison of transfer curves for the $T L^{+}$marker obtained by blending and by treatment with phage T6. Broth-grown Hfr $R 4$ and $\mathrm{F}^{-}: T L-M^{-}$cells were mixed in broth. Five minutes later the mixture was diluted 1000 -fold into broth and into minimal medium. At intervals one sample from each diluted suspension was blended and another treated with phage T6. A large volume of the suspension was treated with phage T6 at 30 minutes, and samples from it were blended at intervals (dotted line).

$$
\begin{aligned}
& \mathrm{O}=\text { minimal medium, blended } \\
& \bullet=\text { minimal medium, T6 treated } \\
& \Delta=\text { broth, blended } \\
& \Delta=\text { broth, T6 treated. }
\end{aligned}
$$

the fall in the curve obtained by blending could commence before all transfer of the $T L^{+}$marker was completed, since it begins when the amount of withdrawal in the interval between successive samples exceeds the increase due to intervening transfer. On the other hand, the curve obtained by $\mathrm{T} 6$ treatment will only reach a maximum when all transfer has taken place, since withdrawal occurs in the 
treated samples themselves, and cannot, therefore, displace the maximum. However, it may be seen from the figure that the time is roughly the same, indicating that the rate of withdrawal does not in fact exceed the rate of transfer until essentially all transfer has taken place. Furthermore, this time is the same as the time when the curves for transfer in broth reach a plateau. This suggests that the extent of the variable delay in the initiation of transfer is the same whether the cells are diluted into minimal medium or into broth after effective contact formation.

The occurrence of withdrawal in samples treated with $\mathrm{T} 6$ may be demonstrated directly by a combination of $\mathrm{T} 6$ treatment and blending. The interrupted curve in Fig. 5 shows the results obtained when T6 was added to a suspension at 30 minutes and samples from this suspension were blended at intervals. It may be seen that if a sample was blended immediately after the addition of T6 the same number of recombinants was formed as in samples without $\mathrm{T} 6$ blended at the same time. When blending was delayed for increasing intervals the number of recombinants fell progressively until finally, 40 minutes after the addition of $\mathbf{T} 6$, the number of recombinants recovered after blending was no greater than without blending. This curve therefore clearly represents the time course of the withdrawal taking place after the addition of T6. Comparison of the rate of withdrawal under these conditions with that occurring in the absence of $\mathbf{T} 6$ as given by the curve for blended samples shows that withdrawal after adsorption of $\mathrm{T} 6$, although it does not differ in extent, is slightly more rapid than without T6.

Considering now the results obtained for transfer in broth, it may be seen from Fig. 5 that the curves obtained for T6 treatment and blending do not differ markedly. In neither is there any fall, and the final number of $T L^{+}$recombinants obtained is about one-quarter of the maximum number obtained in minimal medium. In samples taken at early times the number of recombinants formed from blended samples is perhaps somewhat greater than from the corresponding T6-treated samples, but by 30 minutes there is no longer any difference between the two curves. Since the excess of recombinants in blended compared to T6treated samples in minimal medium is due to the separation by blending of intact pairs in which withdrawal would otherwise take place, the absence of such an excess in broth except for the early points provides convincing support for the idea that in broth the mating pairs are much less stable than in minimal medium.

\section{DISCUSSION}

The study of transfer in mating pairs formed over a short period has brought to light the existence of several factors which have an important effect on the kinetics of chromosome transfer. These factors are: (1) a variable delay between the time of effective contact formation and the initiation of transfer, (2) a marked effect of the presence of a complete mixture of amino-acids on the stability of pairs involving some donor strains but not others, and (3) the eventual withdrawal, in a proportion of the mating pairs, of the chromosome segment which has penetrated the $\mathrm{F}^{-}$cell. These factors interact in a complex fashion, and are therefore 
difficult to separate in a satisfactory manner. A mathematical analysis of the transfer process is presented in the paper by Symonds (1962). In addition to the factors already mentioned, variation in the rate of transfer by cells of some Hfr strains, but not of others, is indicated by the greater asynchrony in the transfer of markers located at increasing distance from the origin of the donor chromosome.

Two general types of explanation may be advanced to account for the variable delay between contact formation and the initiation of transfer:

1. The delay after contact formation may be due to the time required to synthesize structures necessary for chromosomal transfer. The formation of effective contact is defined experimentally as the formation of a bond between donor and recipient cells which is not broken by diluting. It is possible that such a bond might be formed prior to the synthesis of structures necessary for transfer. The pairs may be held together adequately by some type of surface interaction, or, alternatively, synthesis of the connecting structures may proceed in several stages, not all of which need be complete for the formation of a stable bond. Electron micrographs of mating pairs have shown that donor and recipient cells are connected by structures known as conjugation tubes, through which the donor chromosome is thought to be transferred (Anderson, Wollman \& Jacob, 1957).

2. The delay may be due to the fact that transfer can only be initiated at a particular stage in the division cycle of the donor cell. The variable delay would then reflect the asynchrony of the population of donor cells, and consequent variation in the time elapsing before the critical stage is reached. Of particular interest is the possibility that transfer can only begin at a certain point in the DNA (chromosome) replication cycle.

Unfortunately, neither explanation can account satisfactorily for the results available at the present time. There is evidence that some form of synthetic activity is required for effective contact formation. Thus Fisher (1957) has shown that a source of energy is required for contact formation as defined above, as well as during transfer itself. Unpublished experiments (de Haan \& Gross) suggest that this energy is used for protein synthesis by the donor cells, since starvation of the $\mathrm{Hfr}$ culture, but not of the $\mathrm{F}^{-}$, of required amino-acids prevents contact formation, and chloramphenicol has a similar effect. This indicates that specific pairing between donor and recipient cells is not sufficient for contact formation, but that synthesis by the Hfr cells of some type of connecting structure is necessary. However, if the cells are brought into contact with chloramphenicol immediately after contact formation, then no significant inhibition of transfer is observed. It therefore appears likely that once contact formation has occurred, no further protein synthesis is required for transfer to take place. If this is so, then the formation of structures required for transfer must be complete at the time of contact formation, or very shortly after.

Evidence relating the transfer delay to the cellular division cycle is ambiguous. On the one hand the extent of the delay does not appear to be closely correlated with the generation time of the cells. Thus, preliminary experiments indicate that 
the delay is not markedly greater in the case of minimal-grown cells transferring in minimal medium than it is for broth-grown cells transferring broth, despite the fact that the generation time of the cells is almost doubled. Even for the brothgrown cells the maximum interval between contact formation and initiation of transfer appears to be only 15 minutes, whereas the generation time of the bacteria is at least 20 minutes. On the other hand, there is some evidence that DNA synthesis is involved either in the initiation of transfer or in transfer itself. For example, irradiation of the Hfr with small doses of ultraviolet light, which have a fairly specific inhibitory effect on DNA synthesis (Kanazir \& Errera, 1956), appears to increase the degree of asynchrony of transfer of any marker, either by slowing down transfer or by increasing the delay before transfer is initiated (Eggertsson \& Gross, unpublished). The best evidence for involvement of DNA synthesis comes from experiments performed by Dr R. H. Pritchard (personal communication) using a thymine-requiring $\mathrm{Hfr}$ strain isolated by the method of Okada, Yanagisawa \& Ryan (1960). When cells are deprived of thymine and mated under the conditions described in the present work, contact formation is efficient, but little or no transfer takes place. The inhibition of transfer in mating pairs formed in the absence of thymine can be released at will by the addition of thymine to the diluted suspension. Further experiments of this nature should help to clarify the relationship between the transfer delay and DNA synthesis.

Spontaneous interruption of transfer during the oriented penetration of the Hfr chromosome into the recipient cell has been clearly demonstrated by experiments of Wollman \& Jacob (1958). As a result there exists a gradient in the extent of transfer of markers located at increasing distance from the origin of the $\mathrm{Hfr}$ chromosome. The same authors (Jacob \& Wollman, 1958) have shown that the probability of interruption of transfer can be reduced by eliminating as far as possible mechanical stresses on the mating pairs during aeration, dilution and plating. In the present work we have shown that in addition to breaks due to such mechanical causes, the stability of the mating pairs may also be markedly affected by the metabolic activity of the Hfr cells. Thus the presence of a complete mixture of amino-acids in the medium affects the cells of the donor strain in such a way as to reduce the stability of the mating pairs and hence the average duration of chromosomal transfer. As a result the gradient of transfer of markers at increasing distance from the origin of the Hfr chromosome is increased. The extent of the effect of amino-acids depends greatly on the donor strain. Even closely related strains may be quite different in their sensitivity. Thus Hfr $H$ is relatively unaffected by the presence of amino-acids, while $\mathrm{Hfr} C$, which is derived from the same $\mathrm{F}^{+}$strain, is highly sensitive. The same difference exists between the two Hfr strains $R 1$ and $R 4$, despite the fact that they were isolated in the same series of experiments. Little can be said at the moment concerning either the mechanism of action of the amino-acids, or the difference in sensitivity between donor strains. Since all amino-acids are required, the effect is probably related to some form of protein synthesis, possibly breakdown and renewal of the cell wall and structures connecting the mating cells. The difference in sensitivity between Hfr strains is 
not correlated with any microscopically visible variation in cell motility. It may be due to differences in the nature of the conjugation tubes which are synthesized.

It appears unlikely that the chromosome withdrawal which has been demonstrated is due to some type of active reversal of transfer. More probably it is an event which occurs at the time when the mating cells separate. The reasons for thinking this are first that the extent of withdrawal observed is independent of the rate of separation of pairs and hence of the average length of time that the cells remain in contact. Thus, when broth is added to the cells at the time that transfer of the $T L^{+}$marker is completed, and the subsequent rate of separation of the pairs is accelerated, the extent of the fall in number of $T L^{+}$recombinants is unaltered, whereas the rate of the fall is increased. This is what would be expected if withdrawal occurred at the time of separation. If separation and withdrawal were unconnected, one would expect just the reverse result, namely that the extent of withdrawal would be reduced and the relative rate of withdrawal unchanged. Secondly, the adsorption of $\mathrm{T} 6$ to the Hfr cell arrests transfer, but does not prevent withdrawal. If withdrawal depended on the same type of mechanism as transfer, then both processes should be equally affected.

The simplest picture of the withdrawal process, though not necessarily the correct one, is as follows: when transfer is interrupted, either spontaneously or artificially, in such a way that the mating cells separate without a break occurring in the chromosome which is being transferred, then the segment which has penetrated the $\mathrm{F}^{-}$cell may be pulled out when the $\mathrm{Hfr}$ and $\mathrm{F}^{-}$cells move apart. The occurrence of withdrawal in any particular pair should, therefore, depend on whether or not the chromosome is broken at the time when transfer is interrupted. This is clearly so for the treatments employed to interrupt transfer artificially, since withdrawal can occur after $\mathrm{T} 6$ treatment, but not after blending. The violent stresses to which the cells are exposed during blending may be supposed to separate the pairs by breaking through the conjugation tubes connecting the cells and also through the segment of donor chromosome passing through the tube at that time. Due to the break in the chromosome, the segment which has penetrated the $\mathrm{F}^{-}$ cell cannot be withdrawn. T6 treatment, on the other hand, arrests transfer but has no direct effect on the integrity of the conjugation tube or of the chromosome which is being transferred. The evidence indicates that after infection of the $\mathrm{Hfr}$ cell with T6, separation of the pairs occurs for the most part as in the untreated suspension. However, withdrawal which is otherwise unaffected appears to be somewhat more rapid than in the untreated pairs. Spontaneous interruption of transfer must take place without chromosome breakage in a high proportion of the mating pairs in minimal medium, since a considerable fall is observed. On the other hand, the results of the analysis presented by Symonds (1962) indicate that there is little or no withdrawal associated with the accelerated separation of pairs in the presence of amino-acids. Whether this absence of withdrawal is due to chromosome breakage at the time of interruption of mating, or to some other cause, is not known.

The extent of withdrawal as measured by the fall in number of recombinants 
after transfer of a given marker is completed varies very considerably in different experiments, and appears to depend to some extent on the Hfr strain employed. Thus the fall in number of $T L^{+}$recombinants is generally more marked with Hfr $R 4$ than with Hfr $H$ (compare Figs. 3 and 4). The extent of the observed fall due to withdrawal will depend on a variety of factors, such as the degree of synchrony of transfer, the stability of the mating pairs, the extent of breakage due to mechanical causes, as well as on the actual probability of withdrawal in a mating pair in the absence of any break in the chromosome. It may be noted, however, that a fall of at least $50 \%$ in the number of $T L^{+}$recombinants was generally observed in the cross involving $\mathrm{Hfr} R 4$. This fall takes account only of the withdrawal of the $T L^{+}$marker which occurs after all transfer of that marker is complete. Any such withdrawal taking place concurrently with transfer during the first 30 minutes after mixing is not observed. Consequently, it may be concluded that withdrawal occurs in at least half, and probably considerably more, of the mating pairs. It is of some interest to inquire whether the probability of withdrawal of a marker depends on its position with respect to the origin of the $\mathrm{Hfr}$ chromosome. In the case of $\mathrm{Hfr} R 4$ this probability appears to be independent of the position, since essentially the same shape of transfer curve is observed for the sequence of markers $P^{+}, T L^{+}$and $M^{+}$. However, in the case of $\mathrm{Hfr} H$, a fall is consistently observed in the number of $T L^{+}$but not of $\mathrm{gal}^{+}$recombinants (Fig. 3). This apparent absence of withdrawal of later markers may be simply an artifact due to other properties of $\mathrm{Hfr} H$, such as the greater degree of asynchrony of transfer of the later marker. On the other hand, it may really reflect the fact that once a segment of sufficient size has penetrated the $\mathrm{F}^{-}$cell, withdrawal no longer takes place.

\section{SUMMARY}

Chromosome transfer has been studied in mating pairs formed during a 5-minute interval, under conditions that prevent the formation of new pairs. It has been found that, despite restriction of the period of effective contact formation, there is a considerable spread in the time at which transfer is initiated in the different mating pairs. In some pairs there is a delay of as much as 15 minutes before transfer commences.

The presence of broth in the medium in which transfer takes place leads to a marked reduction in the stability of pairs involving some Hfr strains, but has little or no effect on pairs involving others. The action of broth can be ascribed to the effect of a complete mixture of amino-acids on the metabolic activity of the donor cells.

A segment of chromosome which has penetrated from the Hfr into the $\mathrm{F}^{-}$cell may be subsequently withdrawn. Withdrawal may take place in more than half of the mating pairs, and probably occurs at the time when the donor and recipient cells separate. It is prevented if the chromosome breaks when conjugation is interrupted. 
The authors are very grateful to $\mathrm{Dr} W$. Hayes for advice and criticism during the course of this work, and to Dr N. Symonds for very helpful discussion during the preparation of the manuscript. One of us (P. G. de Haan) wishes to express his appreciation for the hospitality extended to him by Dr W. Hayes and the Medical Research Council, and to the Netherlands Organization for the Advancement of Pure Research (Z.W.O.) for a grant.

\section{REFERENCES}

Anderson, T. F., Wolmman, E. L. \& JACOB, F. (1957). Sur les processus de conjugaison et de recombinaison chez Escherichia coli. III. Aspects morphologiques en microscopie électronique. Ann. Inst. Pasteur, 93, 450-455.

Cavalli, L. L. (1950). La sessualità nei batteri. Boll. Ist. sieroter. Milano, 29, 281-289.

Fisher, K. W. (1957). The role of the Krebs cycle in conjugation in Escherichia coli K-12. J. gen. Microbiol. 16, 120-135.

HAYES, W. (1953). The mechanism of genetic recombination in Escherichia coli. Cold Spr. Harb. Symp. quant. Biol. 18, 75-93.

HAYES, W. (1957). The kinetics of the mating process in Escherichia coli. J. gen. Microbiol. 16, 97-119.

JaCOB, F. \& Wollman, E. L. (1956). Sur les processus de conjugaison et de recombinaison chez Escherichia coli. I. L'induction par conjugaison ou induction zygotique. Ann. Inst. Pasteur, 91, 486-510.

JACOB, F. \& Wollman, E. L. (1957). Analyse des groupes de liaison génétique de différentes souches donatrices d'Escherichia coli K-12. C. R. Acad. Sci., Paris, 245, 1840-1843.

$J_{A C O B}, F$. \& Wollman, E. L. (1958). Sur les processus de conjugaison et de recombinaison génétique chez Escherichia coli. IV. Prophages inductibles et mesure des segments génétiques transférés au cours de la conjugaison. Ann. Inst. Pasteur, 95, 497-519.

J ACOB, F., Wollman, E. L. \& HaYes, W. (1956). Conjugation and genetic recombination in Escherichia coli K-12. Cold Spr. Harb. Symp. quant. Biol. 21, 141-162.

KaNAZIR, D. \& ERRERA, M. (1956). Alterations of intracellular deoxyribonucleic acid and their biological consequence. Cold Spr. Harb. Symp. quant. Biol. 21, 19-29.

LEDERBERG, J. (1950). Methods med. Res. 3, 5-22.

Lederbera, J. \& Lederberg, E. M. (1952). Replica plating and indirect selection of bacterial mutants. J. Bact. 63, 399-406.

Okada, T., Yanagisawa, K. \& RYan, F. J. (1960). Elective production of thymine-less mutants. Nature, Lond., 188, 340-341.

SkaAR, P. D. \& Garen, A. (1956). The orientation and extent of gene transfer in Escherichia coli. Proc. nat. Acad. Sci., Wash., 42, 619-624.

Symonds, N. (1962). The kinetics of chromosone transfer in Escherichia coli: a mathematical treatment. Genet. Res. 3, 273-281.

TATUM, E. L. \& Lederibrg, J. (1947). Gene recombination in the bacterium Escherichia coli. J. Bact. 53, 673-684.

Wollman, E. L. \& J $\mathrm{J}_{\mathrm{ACOB}}$, F. (1958). Sur les processus de conjugaison et de recombinaison chez Escherichia coli. V. Le mécanisme du transfert de matériel génétique. Ann. Inst. Pasteur, 95, 641-666.

Wollman, E. L. \& Jacob, F. (1959). La Sexualité des Bactéries. Paris: Masson. 